\title{
Current Status of the KIT Coaxial-Cavity Long-Pulse Gyrotron and its Key Components
}

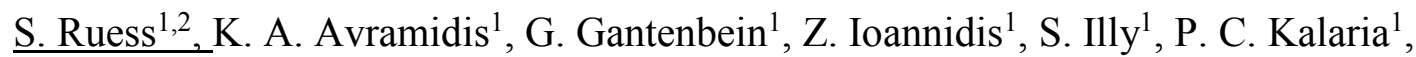 \\ T. Kobarg ${ }^{1}$, I. Gr. Pagonakis ${ }^{1}$, T. Ruess ${ }^{1}$, T. Rzesnicki ${ }^{1}$, \\ M. Thumm ${ }^{1,2}$, J. Weggen ${ }^{1}$ and J. Jelonnek ${ }^{1,2}$
}

${ }^{1} \mathrm{IHM},{ }^{2} \mathrm{IHE}$, Karlsruhe Institute of Technology (KIT), 76131 Karlsruhe, Germany, Sebastian.Ruess@kit.edu

\begin{abstract}
Today's performance requirements for future DEMO gyrotrons are an operating frequency $>200 \mathrm{GHz}$ and an output power of $2 \mathrm{MW}$. A total gyrotron efficiency of better than $60 \%$ must be achieved. Multi-purpose/multi-frequency operation and frequency step-tunability are required also. It has been shown earlier, that the coaxial-cavity technology is a promising candidate [1] for future fusion power plants. In [1] a world record RF output power at 170 $\mathrm{GHz}$ of $2.2 \mathrm{MW}$ has been presented in short-pulse operation ( $\sim$ few milliseconds). However, it has to be proven that the coaxial-cavity technology can be used in steady-state operation. That shall be achieved by upgrading the existing $2 \mathrm{MW} 170 \mathrm{GHz}$ short-pulse prototype with a cooling system and the implementation of advanced Magnetron Injection Guns (MIGs) [3]. Both, the advanced MIGs as well as the longpulse gyrotron will show the way towards a possible solution for DEMO with a significantly higher output power at higher operating frequencies.
\end{abstract}

\section{Bake-out Procedure}

After the significant modification, the gyrotron was baked-out in the in-house oven up to $300{ }^{\circ} \mathrm{C}$. All the components have been designed for a bake-out procedure of more than $400{ }^{\circ} \mathrm{C}$. Especially the solder connections withstand a local temperature of more than $600{ }^{\circ} \mathrm{C}$. In order to record the temperature, 12 thermocouples are connected to the mirror box. The complete bake out procedure has been controlled by a special control system to which a scenario has been programmed. The bake-out scheme consists of three major phases. In the first phase the temperature in the oven is stepwise increased up to the nominal bake out temperature of $300{ }^{\circ} \mathrm{C}$. In the present case, 7 days were spent for the first phase. In the second phase the temperature is kept constant at the nominal value of bake-out for further 7 days. Subsequently, in the third phase, the heating of the oven is switched off and the temperature is decreasing slowly in order to avoid stresses in the components due to different thermal expansions.

The usually occurring event is the increase of vacuum pressure during the ramp-up process. If the pressure exceeds a value of more than $\mathrm{p} 1=1.0 \times 10^{-6}$ mbar the temperature is kept constant until the pressure becomes less than $\mathrm{p} 2=5.0 \times 10^{-7} \mathrm{mbar}$ (marked in Fig. 1 with event 1). If the pressure is smaller than $\mathrm{p} 2$ the oven increases the temperature again. If the pressure is exceeding the pressure level $\mathrm{p} 3=5.0 \times 10^{-5}$ mbar the control stops immediately the heating process and shuts down the oven in a controlled manner (marked in Fig. 1 with event 2).

During the bake-out process the vacuum pressure could be reduced from $2 \times 10^{-7}$ mbar down to $1 \times 10^{-8}$ mbar, which is the lower limit of the vacuum turbo pump.

\section{Alignment of the Coaxial Cavity Insert}

The effective gyrotron operation requires an exact alignment of the mechanical gyrotron axis. I.e. the axis of the electron gun, the axis of the resonator and the axis of the insert with respect to the axis of the magnetic field. Therefore the alignment of the gyrotron axis relative to the axis of the magnetic field has to be satisfied in order to guarantee a contentric electron beam with respect to the cavity and insert. Due to the very tight tolerance requirements it is very important to measure the accuracy of the mentioned gyrotron components at the nominal operation condition, and, if necessary to correct them. The measurement of the electron beam position relative to the insert and cavity wall can be done by the help of the dipole coils of the Oxford Instruments gyrotron magnet at KIT. These coils offers the possibility to shift the electron beam in the xy plane.

In order to measure the position of the hollow electron beam relative to the insert the compression of the magnetic field has to be increased. In this case the electron beam radius is decreasing and moving

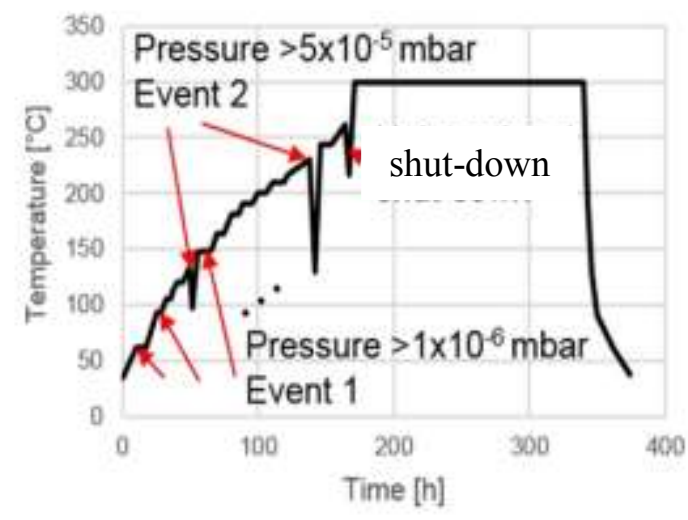

Fig. 1: Temperature profile during tube bake-out. 


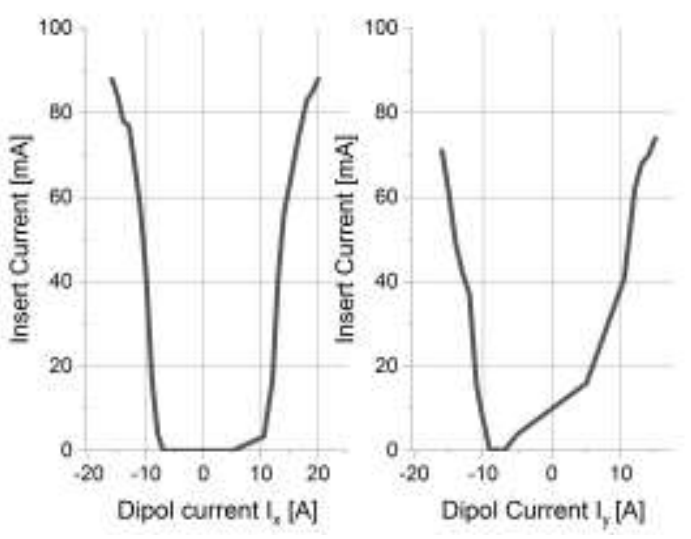

Fig. 2: Measurement of the insert current at different excitations of the dipole currents in xy-plane.

closer to the insert. By the use of the dipole coils the electron beam is touching the insert locally. The current to the insert, measured versus the dipole current, is shown in Fig. 2. In order to prevent damages at the insert, the measurement was done with reduced electron beam parameters. The beam current was $0.335 \mathrm{~A}$ with an acceleration voltage of $1 \mathrm{kV}$. The measurement of the insert current versus the dipol currents in $\mathrm{x}$ - and $\mathrm{y}$ - directions are shown in Fig. 2. Based on the results the deviation between the axis of the electron beam and the axis of the insert is in $\mathrm{x}$-direction $0.036 \mathrm{~mm}$, while the displacement in $\mathrm{y}$-direction is $0.032 \mathrm{~mm}$. The relation between dipole current and shift of the electron beam $\Delta$ rbeam is given by: $\Delta$ rbeam $=0.012 \mathrm{IX}, \mathrm{Y} / \mathrm{A}[\mathrm{mm}]$.

The misalignment, mentioned above, of the insert is negligible and therefore a stable gyrotron operation can be expected.

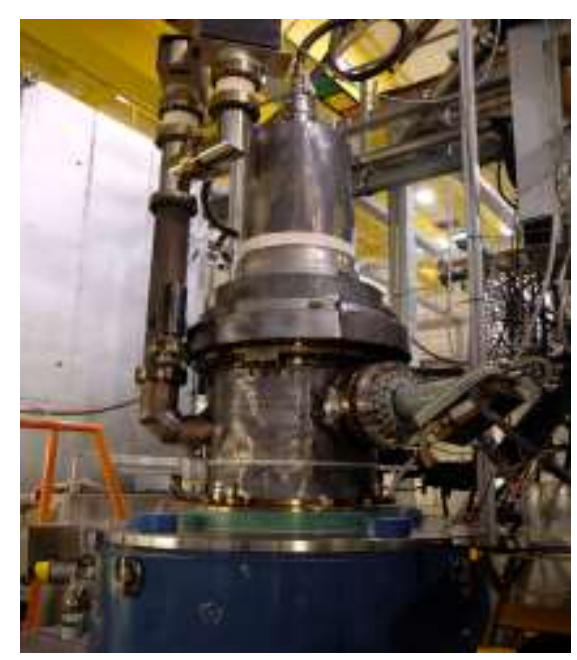

Fig. 3: The coaxial-cavity long-pulse gyrotron in the Oxford Instruments superconducting magnet with the calorimeter for the RF power measurement.

\section{Experimental Verification of the Long-Pulse Coaxial Cavity Gyrotron}

After the bake-out procedure and conditioning of the gyrotron the completely in-house manufactured and assembled components have to be verified and compared with the pre-prototype $2 \mathrm{MW} 170 \mathrm{GHz}$ shortpulse coaxial-cavity gyrotron. For a better comparison, it was decided to use the configuration of the new long-pulse gyrotron with the already existing MIG of the pre-prototype short-pulse gyrotron. This has the advantage that the performance uncertainty of the MIG can be excluded and the focus can be concentrated to the cavity and RF beam forming components.

In Fig. 3 the coaxial-cavity long-pulse gyrotron [3] is shown in the superconducting magnet (SCM). The measurements and analysis of the RF signal has shown that the gyrotron is operating absolutely stable at a single frequency and therefore in only one cavity mode. The measured mode series is in agreement with the calculated prediction.

At $\sim 85 \mathrm{kV}$ a mode switch from the nominal mode $\mathrm{TE}_{34,19}$ at $169.86 \mathrm{GHz}$ to the neighboring mode $\mathrm{TE}_{33,19}$ at $167.79 \mathrm{GHz}$ takes place. The maximum measured output power at the nominal mode is $2.1 \mathrm{MW}$ with an efficiency of $\sim 30 \%$, which is in conformance with the simulation results. With increasing magnetic compression the pitch factor is increasing and therefore the transverse kinetic energy of the electrons and the RF output power are also increasing. This effect was experimentally verified and the maximum measured $\mathrm{RF}$ output power is $2.2 \mathrm{MW}$ with an efficiency of $33 \%$. A further RF power increase is not limited by the gyrotron technology itself. The main limitation is the capability of the SCM and power supply. The new longer-pulse gyrotron has a similar performance as the short-pulse pre-prototype gyrotron, however the efficiency is $3 \%$ higher.

\section{Acknowledgements}

Part of this work is carried out within the framework of the EUROfusion Consortium and is receiving funding from the Euratom research and training programme 2014-2018 under grant agreement No 633053. The views and opinions expressed herein do not necessarily reflect those of the European Commission.

\section{References}

1. T. Rzesnicki, et al., IEEE Trans. Plasma Science, Vol. 38, No. 6, pp. 1141-1149, 2010.

2. S. Ruess, et al., „An Inverse Magnetron Injection Gun for the KIT 2-MW Coaxial-Cavity Gyrotron“, IEEE Transactions on Electron Devices, (63), p. 2104-2109, 2016, DOI:10.1109/TED.2016.2540298.

3. S. Ruess, et al., "KIT coaxial gyrotron development: From ITER toward DEMO". International Journal of Microwave and Wireless Technologies, 1-9. doi:10.1017/S1759078718000144 